\title{
EVALUACIÓN DE LA PROGRAMACIÓN DEL RIEGO EN UNA COMUNIDAD DE REGANTES MEDIANTE BALANCE DE ENERGIA CON IMÁGENES LANDSAT 8
}

\author{
Jiménez-Bello, M.A. (1)(P), Martínez Alzamora, F. (2), Martínez Gimeno, M.A. (3),Intrigliolo, \\ D.S. (4)
}

${ }^{1}$ Investigador de la Universitat Politècnica de València, Instituto Universitario de Ingeniería del Agua y del Medio Ambiente, Camino de Vera, s/n 46022 Valencia. mijibar@dihma.upv.es

${ }^{2}$ Catedrático de la Universitat Politècnica de València, Instituto Universitario de Ingeniería del Agua y del Medio Ambiente, Camino de Vera, s/n 46022 Valencia. fmartine@hma.upv.es

${ }^{3}$ Becaria Formación Profesorado Universitario. Centro de Edafología y Biología Aplicada del Segura. Consejo Superior de Investigaciones Científicas, Campus Universitario de Espinardo 30100, Espinardo, Murcia mamargi8@etsia.upv.es

${ }^{4}$ Científico Titular. Centro de Edafología y Biología Aplicada del Segura. Consejo Superior de Investigaciones Científicas, Campus Universitario de Espinardo 30100, Espinardo, Murcia dintri@cebas.csic.es

\section{Resumen}

El modelo para para el cálculo de la evapotranspiración (ET) por balance de energía a nivel del suelo (SEBAL), una vez ha sido parametrizado para cítricos (Jiménez-Bello et al. 2015), ha sido aplicado a una comunidad de regantes durante la campaña de riego 2014. Entre las parcelas regadas se han seleccionado 22 y se han comparado mensualmente los ratios de la $E T$ calculada mediante la metodología $F A O\left(E_{F A O}\right)$, que lo hace para un cultivo sin limitaciones de agua en el suelo, y la ET calculada con SEBAL (ET SEBAL) frente al ratio de $\mathrm{ET}_{\mathrm{FAO}}$ y los volúmenes aportados a cada parcela (VOL).

Se ha determinado la tendencia lineal y potencial de la comparación de $\mathrm{ET}_{\mathrm{SEBAL}} \mathrm{ET}_{\mathrm{FAO}^{-1}}$ con $\mathrm{ET}_{\mathrm{FAO}} \mathrm{Vol}^{-1}$. En todos los meses ha existido correlación positiva de los datos para las dos tendencias, lo que viene a demostrar que $\mathrm{ET}_{\text {SEBAL }}$ en cultivos estresados es menor que $\mathrm{ET}_{\mathrm{FAO}}$. El modelo potencial ha mostrado mejores coeficientes de determinación, puesto que en cultivos sobreregados llega un límite en el que no se produce más ET y al percolar el agua, ésta no queda recogida en el balance de energía superficial.

A falta de una investigación más detallada de cómo puede afectar el tamaño y la cubierta vegetal de la parcela, SEBAL es una metodología que puede ser utilizada para la estimación de las necesidades de riego en cítricos en comunidades de regantes.

\section{Abstract}

Surface Energy Balance Algorithms for Land (SEBAL) parametrized for citrus (Jiménez-Bello et al. 2015) was applied to an irrigation district during the irrigation season 2014. 22 irrigated orchards were selected and monthly ratios of ET calculated by FAO methodology (ET FAO $_{\text {), }}$ with no soil water limitations, and calculated ET by SEBAL (ET ratio of $\mathrm{ET}_{\mathrm{FAO}}$ and supplied volumes to each orchard (VOL). 
Linear and potential trends of $\mathrm{ET}_{\mathrm{SEBAL}} \mathrm{ET}_{\mathrm{FAO}}{ }^{-1}$ compared to $\mathrm{ET}_{\mathrm{FAO}} \mathrm{VOl}^{-1}$ were determined. In every month there was a positive correlation for the two trends, showing that $\mathrm{ET}_{\mathrm{SEBAL}}$ in stressed crops is lower than $\mathrm{ET}_{\mathrm{FAO}}$. The potential trend has shown best determination coefficients, since for overirrigated crops water percolates and is not registered in the surface energy balance model.

Instead of further research is needed to know how pixel size and vegetation cover affect $\mathrm{ET}_{\text {SEBAL, }}$ SEBAL is a methodology that can be used to estimate citrus water requirements for large irrigation districts,

\section{Introducción}

La evapotranspiración (ET) es un componente clave del balance hidrológico. Desde el punto de vista del uso del agua en la agricultura, la determinación precisa de la ET de un cultivo es fundamental para hacer una gestión eficiente.

Diversas herramientas y modelos para la estimación de la ET han sido propuestos (Rana y Katerji 2000). Entre ellos, el más empleado es el formulado por la FAO (Allen et al. 1998), el cual tiene en cuenta variables climáticas incluidas en la evapotranspiración de referencia $\left(E T_{0}\right)$ y el tipo de cultivo contemplado en un coeficiente de cultivo $\left(\mathrm{K}_{\mathrm{c}}\right)$. La estimación de la evapotranspiración del cultivo $\left(\mathrm{ET}_{\mathrm{c}}\right)$ se calcula como el producto de $\mathrm{ET}_{\mathrm{o}}$ por $\mathrm{K}_{\mathrm{c}}$. Sin embargo, en cultivos altos y con una cubierta vegetal discontinua con un alto grado de acoplamiento con la atmósfera, $\mathrm{K}_{\mathrm{c}}$ puede cambiar dependiendo de las condiciones climáticas locales (Annandale y Stockle 1994; Ballester et al. 2013) y la cantidad de vegetación, altura y densidad (Allen y Pereira 2009). Es por ello importante determinar la posibilidad de usar otras alternativas para estimar la $\mathrm{ET}_{\mathrm{c}}$ de un cultivo leñoso.

La determinación de la transpiración de un árbol mediante sensores de flujo de savia ha sido uno de los métodos más utilizados (Dragoni et al. 2005; Ballester et al. 2013). Además, esta técnica sólo permite determinar el uso de agua de un limitado número de árboles (Smith y Allen 1996). Por lo tanto existen muchas incertidumbres cuando se extrapola la información puntual de un solo árbol a grandes áreas, debido a la gran variabilidad existente entre árboles de una misma parcela comercial. Para extrapolar información puntual a otras escalas como parcelas, zonas de riego o cuencas hidrográficas, se requiere de información espacial. Diferentes metodologías basadas en información obtenida mediante sensores transportados en distintas plataformas se han utilizado para el cálculo de la $\mathrm{ET}_{\mathrm{c}}$ (Kalma et al. 2008).

Una de las metodologías utilizadas para el cálculo de la ET mediante teledetección es determinar el calor latente como un componente residual del balance de energía que se produce a nivel del suelo. Para ello se calcula el calor sensible a partir de la temperatura radiométrica de la superficie obtenida de la banda térmica de las imágenes.

Moran et al. (1994) y Gillies et al. (1997) estudiaron la relación entre la superficie de la temperatura y el Índice Normalizado de Vegetación (NDVI), dado que la transpiración depende de la cantidad de cubierta vegetal. Kustas y Normam (1996) concluyeron que las metodologías más prometedoras era la combinación de la temperatura superficial y de los índices de vegetación o aquellos modelos que acoplaban el de la capa límite atmosférica junto la variación de la temperatura superficial.

Bastiaanssen et al. (1998) desarrollaron Surface Energy Balance Algorithms for Land (SEBAL), un algoritmo calibrado que utiliza imágenes de satélite mediante el modelado 
inverso de dos condiciones extremas (pixel caliente y pixel frío) y que realiza estimaciones del flujo de calor sensible y elimina desviaciones sistemáticas producidas en la estimación de la radiación neta, del flujo de calor del suelo, la temperatura radiométrica y de la resistencia aerodinámica del aire.

La aplicación de SEBAL requiere la utilización de sensores que dispongan de banda en el infrarrojo térmico. Sin embargo, los satélites que incorporan este tipo de tecnología tienen una resolución espacial muy pequeña para la gestión del riego, especialmente en zonas dónde el tamaño de la parcela es reducido como ocurre en algunas zonas del mediterráneo de España. Además cuando el paso de los satélites es poco frecuente, la disponibilidad de las imágenes queda restringida a estas fechas, siempre que las condiciones de nubosidad lo permitan.

Otra limitación es que la $E T$ instantánea ( $\left.E T_{\text {inst }}\right)$, obtenida cuando el satélite pasa, tiene que ser extrapolada a valores diarios para poder emplear la información obtenida para la programación del riego en parcela. El escalado a valores diarios se realiza generalmente asumiendo la conservación de un factor de escala, como la fracción evaporativa (Crago y

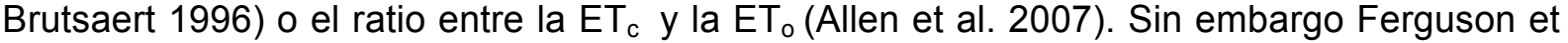
al. (2010) identificaron la suposición de este ratio como constante, como la mayor fuente de incertidumbre en la estimación de flujos de energía mediante teledetección, particularmente para sistemas agrícolas con un bajo grado de acoplamiento con la atmósfera alrededor existente (Jarvis 1985). Este es el caso de los cítricos, un cultivo perenne que regula los estomas bajo cambios en las condiciones medioambientales, fundamentalmente por el déficit de presión de vapor, resultando en un $\mathrm{K}_{\mathrm{c}}$ variable a lo largo del día (Kriedemann 1986).

En este trabajo se presenta la aplicación de la parametrización de SEBAL para cítricos durante una campaña de riego, previamente validada comparando los resultados con los obtenidos a partir de una torre de Covarianza de Torbellinos (Jiménez-Bello et al 2015), en una comunidad de regantes de la provincia de Valencia. Las imágenes empleadas han sido del satélite Landsat 8.

\section{Metodología}

\subsection{Caso de estudio}

El caso de estudio es el Sector XI de Picassent en Valencia (España; 39 22' 43" N, $0^{\circ} 28^{\prime}$ 20 " W)) donde en la actualidad se riegan 307 ha, en su mayoría cítricos, con un tamaño medio de las parcelas de $3600 \mathrm{~m}^{2}$. La Figura 1 muestra el caso de estudio compuesto por dos redes de distribución que funcionan de manera independiente abastecidas por una balsa de regulación. 

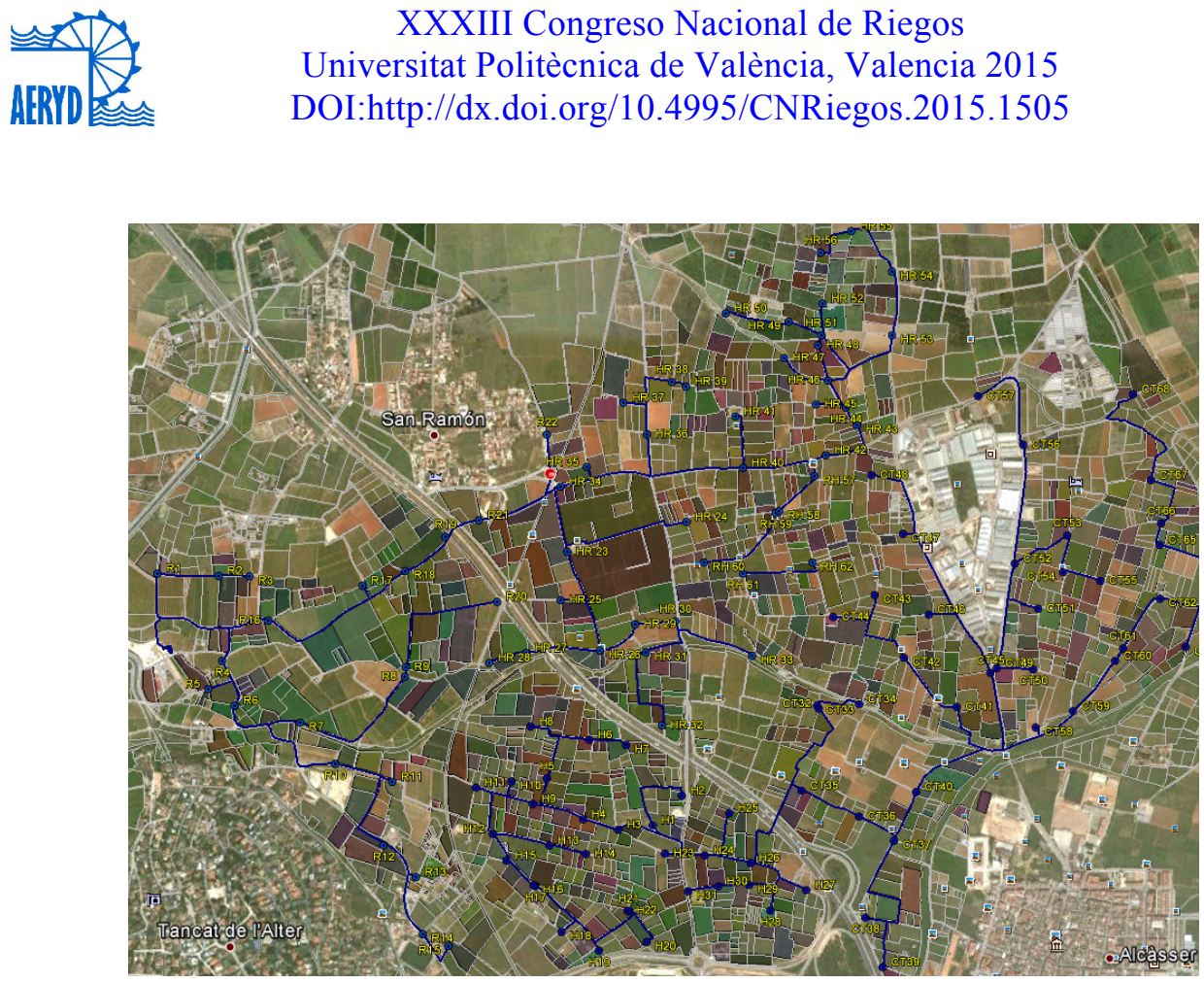

Figura 1 Sector XI, Picassent (Valencia)

14 imágenes de las 28 disponibles desde el mes de Abril a Noviembre fueron procesadas mediante la aplicación de SEBAL al estar libres de nubes en la zona de estudio (Figura 2).

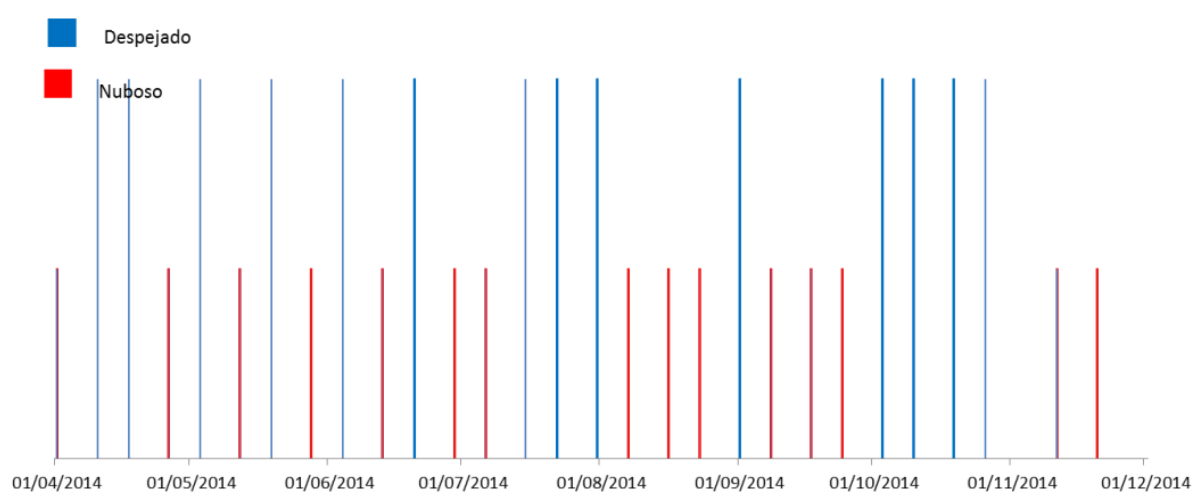

Figura 2 Imágenes disponibles entre los meses de Abril y Noviembre de 2014 donde se indica la nubosidad en la zona de estudio.

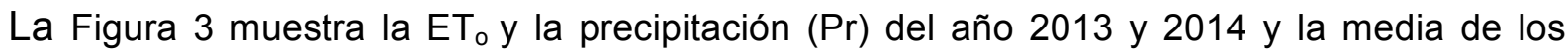
últimos 10 años. 


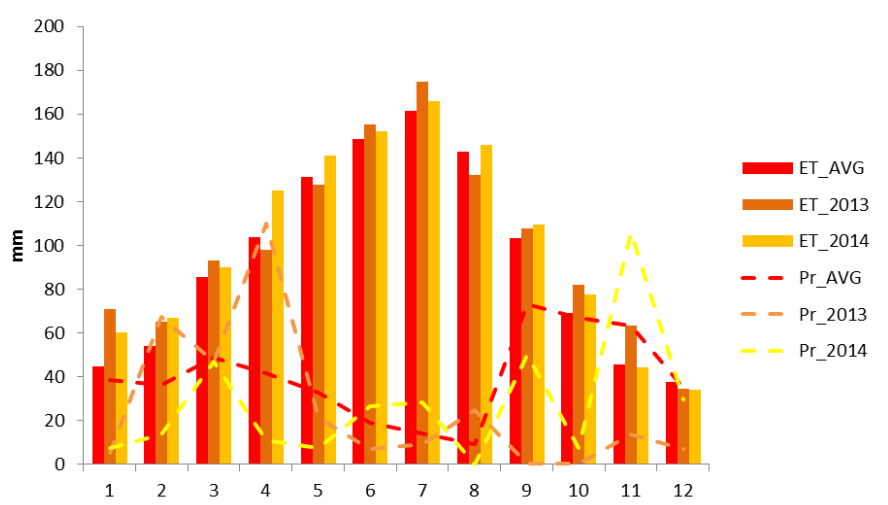

Figura 3 Evapotranspiración de referencia (ET_AVG, ET_2013; ET_2014) y precipitación (Pr_AVG, Pr_2013; Pr_2014) para los últimos 10 años y los años 2013 y 2014.

\subsection{Cálculo de necesidades de riego mediante SEBAL}

SEBAL calcula ET como el residuo de la ecuación del balance de energía

$$
L E=R_{n}-G-H
$$

donde LE es el calor latente de la energía consumida por la evapotranspiración, $R_{n}$ es la radiación neta, $\mathrm{G}$ es el calor sensible transmitido al suelo y $\mathrm{H}$ es el calor sensible transmitido por calor al aire, todo en $\mathrm{W} \mathrm{m}^{-2}$. $\mathrm{R}_{\mathrm{n}}$ fue calculado como en Allen et al. (2011). Para el cálculo de $G$ fue adoptado la formula empírica propuesta por Bastiaansen (2000), la cual estima el ratio $\mathrm{G}_{\mathrm{n}}{ }^{-1}$ próximo al medio día como:

$$
\frac{G}{\mathrm{R}_{\mathrm{n}}}=\frac{\mathrm{T}_{\mathrm{s}}}{\alpha}\left(0.0038 \cdot \alpha+0.0074 \cdot \alpha^{2}\right) \cdot\left(1-0.98 \cdot N D V I^{4}\right)
$$

donde $T_{s}$ es la temperatura de la superficie $(K)$ y $\alpha$ es el albedo superficial

H se calcula a partir de una ecuación aerodinámica

$$
H=\frac{\left(\rho \cdot c_{p} \cdot d T\right)}{r_{a h, 1,2}}
$$

donde $\rho$ es la densidad del aire $\left(\mathrm{kg} \mathrm{m}^{-3}\right), \mathrm{C}_{\mathrm{p}}$ es el calor específico del aire a presión constante $\left(1004 \mathrm{~J} \cdot \mathrm{kg}^{-1} \cdot \mathrm{K}^{-1}\right)$ y $\mathrm{r}_{\mathrm{ah} 1,2}$ es la resistencia aerodinámica $\left(\mathrm{s} \mathrm{m}^{-1}\right)$ entre dos alturas cercanas a la superficie, $\mathrm{z}_{1}$ y $\mathrm{z}_{2}$ (generalmente 0.1 y $2 \mathrm{~m}$ ) sobre la altura del plano de desplazamiento 0 calculada como la función de la resistencia aerodinámica para cada uno de los píxeles de forma individual. dT $(\mathrm{K})$ es la diferencia de temperaturas entre dos alturas $z_{1}$ y $z_{2}$ (Allen et al. 2011). dT se asume que es linear y proporcional a $T_{s}$ como Bastiaanssen (1995) empíricamente probó:

$$
d T=b+a \cdot T_{s}
$$

Puesto que existen dos variables desconocidas en (3), dt y $r_{a h}$, se lleva a cabo un proceso de calibración interna. Con este fin se seleccionan dos píxeles extremos que representan una superficies agrícola muy seca y húmeda, respectivamente (Allen et al., 2011). 
Como píxel frío se seleccionó un lago cercano, dónde se asumió que dT era 0 , suponiendo que la temperatura del aire era igual a $T_{s}$ y $H$ era 0 . El límite superior de la función dT se estima a partir de un pixel caliente donde se asume que el suelo está lo suficientemente seco de forma que $L E=0$.

$d T_{\text {hot }}=\rho_{\text {airhot }} \cdot C_{p} \frac{\left(R_{n}-G\right)}{r_{\text {ahhot }}}$

donde $r_{a n h o t}$ es $r_{a h}$ calculada para la rugosidad y condiciones de estabilidad del pixel caliente. La selección del pixel caliente fue realizada manualmente por medio de ortofotos de alta resolución. El pixel caliente fue cambiando a lo largo de la sesión si la presencia de vegetación era detectada mediante el análisis del valor del NDVI.

$r_{\text {ah }}$ ha sido calculada para las parcelas de cítricos aplicando una relación entre la longitud de la rugosidad para la transferencia del momento $\left(\mathrm{z}_{\mathrm{om}}\right)$ y la altura del cultivo (h) de 0,19 obtenida a partir de datos de Covarianza de Torbellinos (Jiménez-Bello et al. 2015). La altura de los cultivos se ha estimado mediante LIDAR, relacionándola con la cubierta vegetal (GC) obtenida mediante ortofotos correspondientes en el tiempo (Jiménez-Bello et al 2012). Posteriormente se ha remuestrado al tamaño de pixel de Landsat 8 tomando la media más la desviación estándar. Como pixel frío se ha se seleccionado un lago próximo. La evapotranspiración instantánea ha sido escalada mediante dos métodos:

a) El coeficiente de cultivo calculado en el momento de la toma de la imagen $\left(\mathrm{Kc}_{\text {inst }}\right)$ se mantiene constante a lo largo del día.

$$
K c_{\text {inst }}=\frac{E T_{\text {inst }}}{E T_{\text {oinst }}}
$$

b) La relación entre la evapotranspiración instantánea y la radiación observada $\left(R_{s}\right)$ cuando pasa el satélite, es la misma que la evapotranspiración de cultivo y la radiación observada diaria.

$$
E T_{\text {SolRad }}=\frac{1}{n} \sum_{d=1}^{n} \frac{1800 \cdot E T_{\text {inst }}\left(t_{d}\right)}{S F_{d}\left(t_{d}\right)}
$$

Tomando como factor de escala $\left(\mathrm{SF}_{\mathrm{d}}\right)$

$$
S F_{d}=\frac{1800 \cdot R_{S}(t)}{\int_{d} R_{S}(t) d t}
$$

Donde 1800 es el número de segundos en 30 minutos

\subsection{Cálculo de necesidades de riego mediante método FAO}

La metodología adoptada fue la propuesta por Castel (2000) en la que se relaciona $\mathrm{k}_{\mathrm{c}}$ con GC tal como se indica en la Tabla 1 . El $\mathrm{K}_{\mathrm{c}}$ obtenido se asume que es para un cultivo sin restricción de agua en el suelo. 
Tabla 1: Coeficiente de cultivo $\left(\mathrm{K}_{\mathrm{c}}\right)$ según la cubierta vegetal $(\mathrm{GC}, \%)$ para cítricos

\begin{tabular}{|c|c|}
\hline GC (\%) & Relación $\mathrm{K}_{\mathrm{c}}$-GC \\
\hline $20>\mathrm{GC}$ & $\mathrm{K}_{\mathrm{c}}=0.021+\mathrm{GC}^{*} 0.0174$ \\
\hline $20<\mathrm{GC}<70$ & $\mathrm{~K}_{\mathrm{c}}=0.274+\mathrm{GC}^{*} 0.005$ \\
\hline $70<\mathrm{GC}$ & $\mathrm{K}_{\mathrm{c}}=\mathrm{K}_{\mathrm{c} 70}$ \\
\hline
\end{tabular}

A su vez $\mathrm{K}_{\mathrm{c}}$ es corregido dependiendo de la época del año como se indica en la Tabla 2.

Tabla 2: Factor de corrección mensual del $\mathrm{K}_{\mathrm{c}}$ dependiente del mes del año.

\begin{tabular}{|l|l|l|l|l|l|l|l|l|l|l|l|l|}
\hline $\begin{array}{l}\text { kc medio } \\
\text { si } \mathbf{A}>\mathbf{7 0}\end{array}$ & \multicolumn{10}{|c|}{$\mathbf{f}_{\text {cmes }}$} \\
\hline $\mathrm{k}_{\mathrm{c} 70}$ & Ene & Febr & Mar & Abr & May & Jun & Jul & Ago & Sep & Oct & Nov & Dic \\
\hline 0.647 & 0,97 & 0,96 & 0,97 & 0,91 & 0,81 & 0,91 & 1,00 & 1,16 & 1,09 & 1,24 & 1,07 & 0,93 \\
\hline
\end{tabular}

GC fue extraída mediante técnicas de análisis de imágenes como se describe en JiménezBello et al. (2012)

\subsection{Validación de los resultados}

Para validar los resultados se han comparado la ET calculada con SEBAL (ET parcelas dónde se han estimado la evapotranspiración $\left(\mathrm{ET}_{\mathrm{FAO}}, \mathrm{mm}\right)$ tal como se ha descrito anteriormente. El grado de estrés hídrico de cada una de las parcelas se ha determinado comparando la $\mathrm{ET}_{\mathrm{FAO}}$ con los volúmenes realmente aportados (Vol, $\mathrm{mm}$ ) mediante el indicador $\mathrm{ET}_{\mathrm{FAO}} \mathrm{Vol}^{-1}$. Cuando $\mathrm{ET}_{\mathrm{FAO}} \mathrm{Vol}^{-1}>1$, se asume que el cultivo está recibiendo menos agua de la requerida, siendo mayor el estrés cuanto mayor es el valor de este indicador. De modo contrario, cuando $\mathrm{ET}_{\mathrm{FAO}} \mathrm{Vol}^{-1}<1$, se asume que el cultivo está recibiendo más agua de la estimada.

Un total de 22 parcelas, desde 0,3 a 3,5 ha, fueron seleccionadas con la garantía de que la superficie registrada estaba medida por el contador asignado. Para los meses de Mayo a Octubre se han comparado estos indicadores para las 22 parcelas y se ha determinado la curva de tendencia asumiendo un modelo lineal y potencial. Los métodos de interpolación de $\mathrm{ET}_{\text {inst, }}$, han sido los descritos en la sección 2.2.

\section{Resultados}

La Figura 4 muestra los resultados de comparar $\mathrm{ET}_{\mathrm{SEBAL}} \mathrm{ET}_{\mathrm{FAO}}{ }^{-1}$ con $\mathrm{ET}_{\mathrm{FAO}} \mathrm{Vol}^{-1}$ para los meses de Mayo a Octubre del año 2014. Los valores interpolados se obtuvieron asumiendo Ec (7). Se observa que para todos los meses existe una tendencia lineal con un coeficiente de determinación $\left(R^{2}\right)$ máximo para el mes de Mayo $\left(R^{2}=0.61\right)$ y mínimo para el mes de Octubre $\left(R^{2}=0.31\right)$. Asumiendo la interpolación de los resultados por el método de escalado del coeficiente de cultivo constante (Ec 6 ), los resultados son similares siendo el mejor $\mathrm{R}^{2}$ para Septiembre $\left(R^{2}=0.56\right)$ y el peor para Octubre $\left(R^{2}=0.40\right)$. Esta linealidad indica que $\mathrm{ET}_{\text {SEBAL }}$ fue menor que $\mathrm{ET}_{\mathrm{FAO}}$ cuando el cultivo estaba estresado y mayor cuando el cultivo estaba regado en exceso, lo que viene a indicar que $\mathrm{ET}_{\text {SEBAL }}$ calcula la evapotranspiración real del cultivo, siendo útil para detectar a nivel de comunidad de regantes qué parcelas están regadas por exceso y cuales por defecto. 

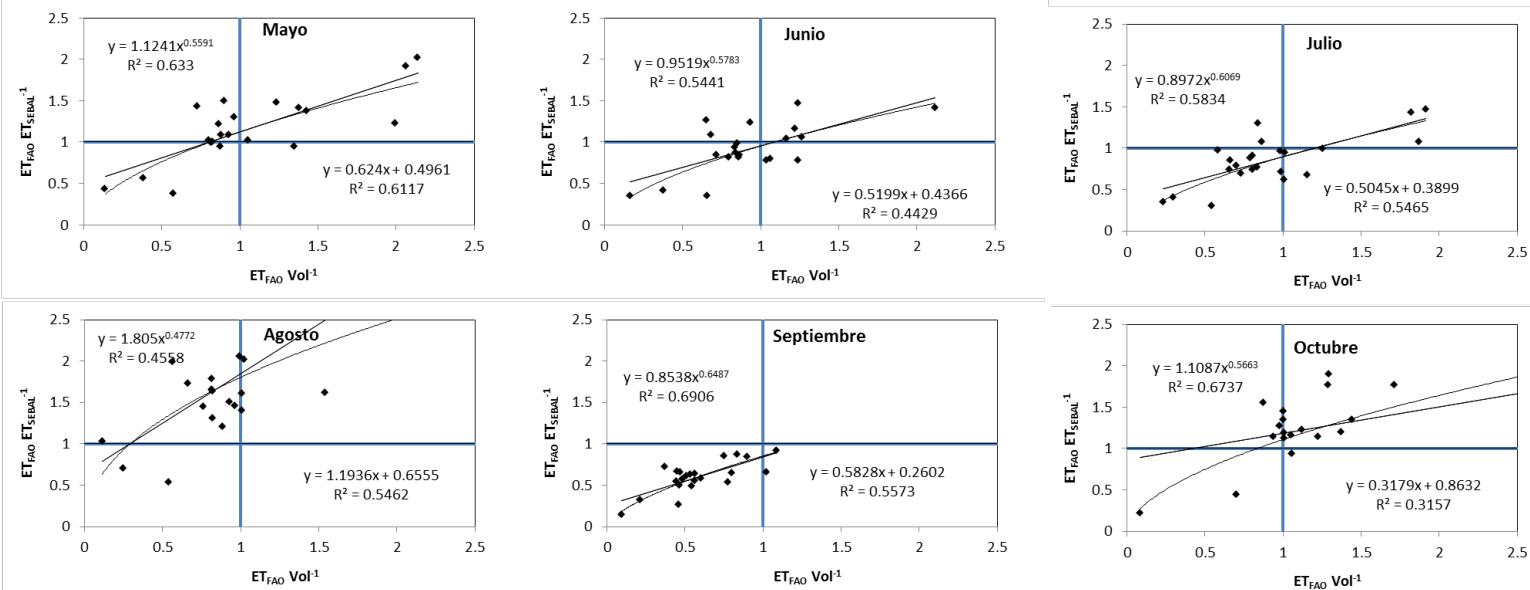

Figura 4 Comparación de $\mathrm{ET}_{\mathrm{SEBAL}} \mathrm{ET}_{\mathrm{FAO}}{ }^{-1}$ con $\mathrm{ET}_{\mathrm{FAO}} \mathrm{Vol}^{-1}$ para los meses de Mayo a octubre de las 22 parcelas seleccionadas. $\mathrm{ET}_{\text {SEBAL }}$ ha sido obtenida asumiendo que la relación entre la evapotranspiración instantánea y la radiación observada cuando pasa el satélite es la misma que la evapotranspiración de cultivo y la radiación observada diaria Los métodos de interpolación mostrados son el lineal y el potencial y el coeficiente de determinación $\mathrm{R}^{2}$.

El modelo potencial muestra en general mejores $R^{2}$ que el lineal con un máximo $R^{2}$ de 0.69 (Septiembre) y 0.75 (Octubre), para los dos métodos de interpolación de la $\mathrm{ET}_{\text {inst }}$ y mínimos de 0.32 (Octubre) y 0.36 (Agosto). Este hecho se debe a que cuando el cultivo está sobreregado $\left(\mathrm{ET}_{\mathrm{FAO}} \mathrm{Vol}^{-1}<0.5\right)$, el agua percola, por lo que no es recogido en el balance energético (Ec 1). De este modo, pese a que $\mathrm{ET}_{\mathrm{FAO}} \mathrm{Vol}^{-1}$ disminuya, $\mathrm{ET}_{\text {SEBAL }}$ permanece constante, lo que justifica que el modelo potencial se ajuste mejor que el lineal. Santos et al. (2010) observaron el mismo fenómeno aplicando una variante de SEBAL (METRIC) en parcelas de olivo.

Se observa que en el mes de Octubre, la mayoría de las parcelas han sufrido estrés y en Septiembre por lo contrario han sido regadas en exceso. Este hecho se puede explicar porque en Septiembre de 2014, la pluviometría fue mayor de lo normal y en Octubre sucedió lo contrario, tal como se observa en la Figura 3. No obstante los factores de corrección experiméntales para el mes de Septiembre y Octubre están determinados para condiciones normales de pluviometría, por lo que se adapta mejor una método de balance de energía como SEBAL, que no está basado en datos empíricos locales.
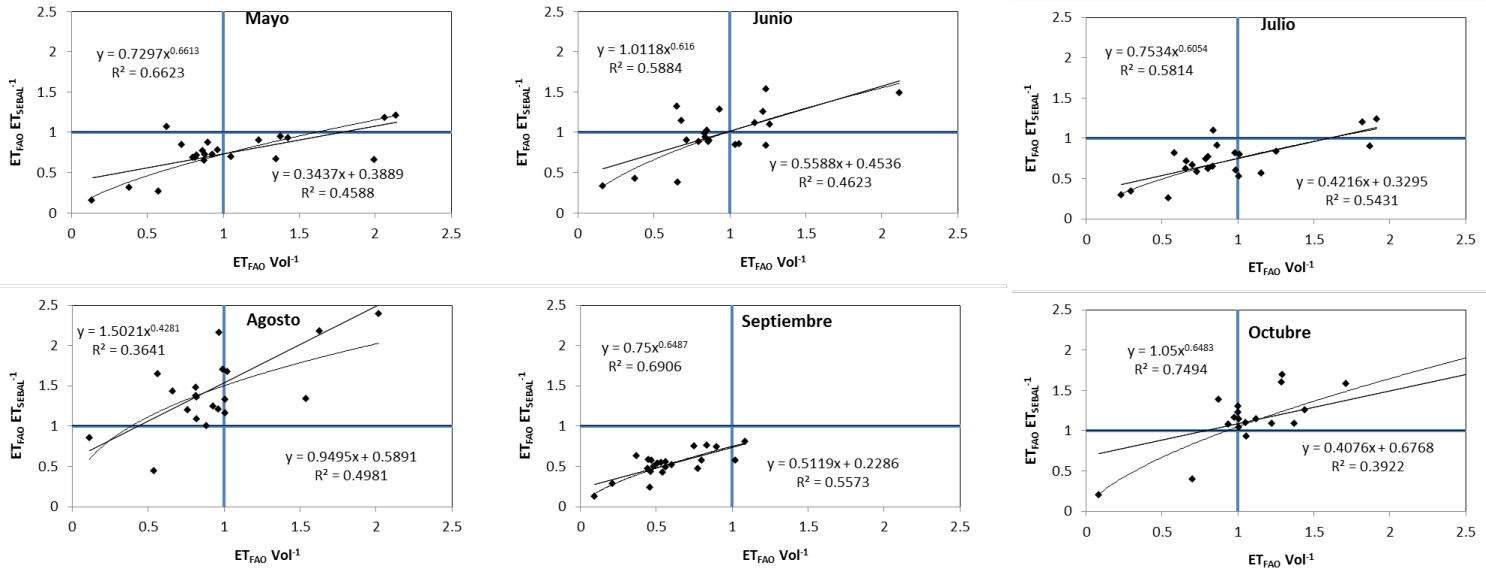

Figura 5 Comparación de $\mathrm{ET}_{\mathrm{SEBAL}} \mathrm{ET}_{\mathrm{FAO}^{-1}}$ con $\mathrm{ET}_{\mathrm{FAO}} \mathrm{Vol}^{-1}$ para los meses de Mayo a octubre de las 22 parcelas seleccionadas. $\mathrm{ET}_{\mathrm{SEBAL}}$ ha sido calculada por el método de escalado del 
coeficiente de cultivo constante. Los métodos de interpolación mostrados son el lineal y el potencial y el coeficiente de determinación $\mathrm{R}^{2}$.

\section{Conclusiones}

A falta de una investigación más detallada de cómo puede afectar el tamaño y la cubierta vegetal de la parcela, SEBAL es una metodología que puede ser utilizada para la estimación de las necesidades de riego en cítricos en comunidades de regantes.

Es capaz de detectar aquellas parcelas que sufren estrés al calcular la evapotranspiración real del cultivo. Junto con los modelos basados en indicadores de vegetación como Castel (2000) y los registros de los contadores de las parcelas, permite la realización de mapas de indicadores de estrés hídrico. Además el cálculo de la ET sobre grandes regiones, como en el caso de la Comunidad Valenciana con 150.000 ha de cítricos, permitiría mejorar el uso del agua del riego.

\section{Agradecimientos.}

Este trabajo ha sido financiado por el proyecto FIGARO del Séptimo Programa Marco europeo. Los autores agradecen la ayuda proporcionada por el personal del Sector XI de Picassent.

\section{Bibliografía}

Allen R G, Pereira LS, Raes D, Smith M (1998) Crop evapotranspiration. Guide lines for computing crop water requirements. FAO Irrigation and Drainage Paper No. 56, Rome, Italy.

Allen R G, Tasumi M, Morse A, Trezza R, Wright J L, Bastiaanssen W, Kramber W, Lorite I J, Robison C W (2007a) Satellite-based energy balance for mapping evapotranspiration with internalized calibration (METRIC)—applications. J. Irrig. Drain. Eng. ASCE 133 (4), 395-406. Annandale J G, Stockle C O (1994) Fluctuation of crop evapotranspiration coefficients with weather. A sensitivity analysis. Irr. Sci. 15,1-7.

Allen R G, Irmak A, Trezza R , Hendrickx J M H, Bastiaanssen W, Kjaersgaard J (2011) Satellite-based ET estimation in agriculture using SEBAL and METRIC. J. Hydrolog. Process. 25,4011-4027.

Ballester C, Castel J, Testi L, Intrigliolo D S, Castel J R (2013) Can heat-pulse sap flow measurements be used as continuous water stress indicators of citrus trees? Irrigat. Sci. DOI: $10.1007 / \mathrm{s} 00271-012-0386-5$.

Bastiaanssen W G M (1995) Regionalization of surface flux densities and moisture indicators in composite terrain: a remote sensing approach under clear skies in Mediterranean climates. PhD Diss., CIP Data Koninklijke Bibliotheek, Den Haag, the Netherlands; 273.

Castel, J.R., 2000. Water use of developing citrus canopies in Valencia, Spain. Proceeding International Society Citriculture, IX Congress:223-226.

Crago R D, Brutsaert W (1996) A daytime evaporation and the self-preserve of the evaporative fraction and the Bowen ratio. J. Hydrol. 178, 241-255. 
Dragoni D, Lakso A N, Piccioni R M (2005) Transpiration of Apple trees in a humid climate using heat pulse sap flow gauges calibrated with whole-canopy gas Exchange chambers. Agr. For. Meteorol. 130,85-94.

Ferguson C. R., Sheffield J., Wood E.F. and Gao H. (2010)

Quantifying uncertainty in a remote sensing-based estimate of evapotranspiration over continental USA, Int J Remote Sens, 31:14, 3821-3865.

Gillies R T, Carlson T N, Cui J, Kustas W P, Humes K S (1997) A verification of the "triangle" method for obtaining surface soil water content and energy fluxes from remote measurements of the Normalized Difference Vegetation Index (NDVI) and surface radiant temperatures. Int. J. Remote Sens. 18 (15), 3145-3166.

Jarvis P G (1985) Coupling of transpiration to the atmosphere in horticultural crops: the omega factor. Acta Horticult. 171, 187-205.

Jiménez-Bello M.Á, Ruiz L.Á, Hermosilla T, Recio J, Intrigliolo D.S (2012). Use of remote sensing and geographic information tools for irrigation management of citrus trees. chapter Use of remote sensing and geographic information tools for irrigation management of citrus trees:147-160; CIHEAM., ISBN: 2-85352-482-5.

Jiménez-Bello M.A., Castel J.R.; Luca T., Intrigliolo D.S. (2015). Assessment of a remote sensing energy balance methodology (SEBAL) using different interpolation methods to determine evapotranspiration in a citrus orchard. IEEE J-STARS. DOI: 10.1109/JSTARS.2015.2418817

Kalma J D, MacVicar T R, McCabe M F (2008) Estimating land surface evaporation:a review of methods using remotely sensed surface temperature data. Surv. Geophys., doi:10.1007/s10712-008-9037-z.

Kustas W P, Li F, Jackson T J, Prueger J H, MacPherson J I, Wolde M (2004) Effects of remote sensing pixel resolution on modeled energy flux variability of croplands in lowa. Remote Sens. Environ. 92,535-547.

Moran M S, Clarke T R, InouemY, Vidal A (1994) Estimating crop water deficit usingthe relation between surface-air temperature and spectral vegetation index.

Remote Sens. Environ. 49, 246-263.

Rana G, Katerji N (2000) Measurement and estimation of actual evapotranspiration in the field under Mediterranean climate: a review. Eur. J. Agron. 13 (2/3), 125-153.

Santos, C, Lorite, IJ, Tasumi, M, Allen, RG, Fereres, E (2010) Performance assessment of an irrigation scheme using indicators determined with remote sensing techniques. Irrig Sci 28: pp. 461-477 